Methods: Five hundred and sixty five (565) children were diagnosed with KD during the period from January 1993 to December 2016 in Pediatric Rheumatology Clinic, Advanced Pediatrics Centre, Postgraduate Institute of Medical Education and Research, Chandigarh, India. Nine children had pulmonary presentation of $\mathrm{KD}$. A retrospective case review with respect to clinical presentation, radiological findings and treatment was done.

Results: Pulmonary presentation of KD was seen in $1.6 \%$ patients. Mean age at diagnosis of KD was 2.9years (range 9 months -4 years). $77.8 \%$ patients had no features suggestive of KD either on history or at presentation. First sign of KD was noted at a mean duration of 17.6 days (range 6-28 days) from the onset of symptoms. Periungual desquamation was the most common clinical sign seen in $66.6 \%$ patients followed by erythematous rash and perianal desquamation in $33.3 \%$ patients each

Persistent fever, thrombocytosis and elevated erythrocyte sedimentation rate (ESR) and C-reactive protein (CRP) were seen in all patients. Microbiological investigations showed evidence of infection in only 2 patients - methicillin sensitive staphylococcus aureus in pus and positive mycoplasma agglutinin titre in one patient each. Parenchymal consolidation was the most common radiological finding $(100 \%)$ followed by pleural effusion $(55.5 \%)$, empyema $(33.3 \%)$ and pneumothorax $(11.1 \%)$. Coronary artery abnormalities were evident on echocardiography in $22.2 \%$ patients with dilatation of right coronary artery and left main coronary artery in 1 patient each.

All patients received intravenous antimicrobials for pneumonia. Intravenous immunoglobulin (IVIG) at $2 \mathrm{~g} / \mathrm{kg}$ was given after a mean duration of 24 days of onset of fever. Fever, which was unresponsive to intravenous antimicrobials, responded to IVIG therapy in all except 2 patients which required a second dose of IVIG. 55.5\% patients required intercostal drainage tube (ICDT) insertion, 2 patients required streptokinase and 1 patient each required video assisted thoracoscopic surgery (VATS) and decortication. Mean follow up period was 15.9 months (range 0-62 months).

Conclusions: Pulmonary involvement in patients with $\mathrm{KD}$ is uncommon and is less commonly recognized. Unresolving pneumonia in a child who continues to be febrile despite adequate antimicrobials with elevated inflammatory markers can be a clue towards the diagnosis of KD. Early recognition can prevent delays in diagnosis and shorten the hospital stay.

Disclosure of Interest: None declared

DOI: 10.1136/annrheumdis-2017-eular.6278

\section{THU0497 RHEUMATOLOGICAL MANIFESTATIONS OF X-LINKED AGAMMAGLOBULINEMIA: PROFILE OF 17 CASES FROM A TERTIARY CARE CENTER IN NORTH INDIA}

A. Gupta, D. Suri, A. Gupta, A. Rawat, S. Singh. Department of Pediatrics, Postgraduate Institute of Medical Education and Research, Chandigarh, India, Chandigarh, India

Background: $X$-linked agammaglobulinemia (XLA) is characterized by recurrent bacterial sino-pulmonary infections. Arthritis can be seen in 10-30\% patients of hypogammaglobulinemia (1) and is usually a mono- or oligoarthritis of large joints, but polyarthritis has also been defined.

Objectives: We describe our experience of managing children with XLA who presented with rheumatological manifestations.

Methods: Sixty two children were diagnosed with XLA during the last 10 years in Primary Immunodeficiency Clinic, Advanced Pediatrics Centre, Postgraduate Institute of Medical Education and Research, Chandigarh, India. Seventeen children had rheumatological manifestations. A retrospective case review with respect to clinical presentation, musculoskeletal findings and treatment was done. Results: Rheumatological manifestations were seen in $27.4 \%$ patients of XLA. Thirteen out of 17 patients had a proven mutation in Btk gene. Mean age at symptom onset was 3.3 years (range 6 months -13 years) and mean age at diagnosis of XLA was 5.6 years (range 1.5- 10 years). Rheumatological manifestations were seen at a mean age of 8.7 years (range 1.5- 20 years). In 2 patients, arthritis preceded the diagnosis of XLA while 10 patients developed rheumatological manifestations after the diagnosis of XLA. Arthritis as an initial presentation of XLA was seen in 5 patients.

Oligoarthritis was the most common presentation seen in 15 patients. Knee was the most commonly involved joint (11 patients) followed by ankle joint in 5 patients and shoulder, wrist and hip arthritis in 2 patients each. One patient each had arthritis involving proximal interphalangeal joints of both hands, clinical evidence of sacroiliitis and spondylodiscitis involving L4-5 and L5-S1 vertebrae. Three patients manifested as septic arthritis (fever, redness, warmth and pus in joint) involving shoulder, ankle and knee joint respectively.

Joint aspiration and/or arthrotomy had been performed in 8 patients, however cultures were sterile in all. Microbiological investigations for mycoplasma infection (viz. culture and polymerase chain reaction) in joint fluid/pus could not be performed due to unavailability. All patients received replacement intravenous immunoglobulin (IVIG - $400 \mathrm{mg} / \mathrm{kg}$ body weight) and co-trimoxazole prophylaxis. Eight patients received macrolide antimicrobials (erythromycin or azithromycin) for clinical suspicion of mycoplasma arthritis.

One patient expired during the course of his illness while four patients were lost to follow up. Arthritis showed clinical improvement after IVIG replacement therapy in remaining 12 patients with resolution of arthritis after first dose in 11 patients and after 6 months of replacement therapy in 1 patient. None of the patients had recurrence of joint symptoms on follow up, however radiological evidence of spondylodiscitis persisted in one patient.

Conclusions: Arthritis is a relatively frequent manifestation in patients with hypogammaglobulinemia. Family history and history of recurrent infections in a child with arthritis may provide a clue towards the diagnosis of primary immunodeficiency. Prompt institution of regular IVIG replacement therapy results in resolution of arthritis and prevents its recurrence.

Disclosure of Interest: None declared

DOI: 10.1136/annrheumdis-2017-eular.6820

\section{THU0498 PFAPA SYNDROME IN LARGE PEDIATRIC POPULATION: A SINGLE CENTER EXPERIENCE}

E. Pehlivan, A. Adrovic, S. Sahin, K. Barut, O. Kul, O. Kasapcopur. Pediatric Rheumatology, Istanbul University, Cerrahpasa Medical School, Istanbul, Turkey

Background: Periodic fever, aphthosis, pharyngitis, and adenitis (PFAPA) syndrome is an auto-inflammatory condition of unknown etiology. It is the second most common auto-inflammatory disease in our country, following familial Mediterranean fever. Previous studies showed that tonsillectomy represents efficient treatment options.

Objectives: Our aim was to explore the main clinical features, response to tonsillectomy and long-term outcome of PFAPA pediatric patients in a single cohort. We assessed association of MEFV gene mutation with disease characteristics and treatment response.

Methods: We reviewed medical records of patients who were diagnosed with PFAPA syndrome between the January 2010 and June 2016. All of the recorded 562 patients were called by the telephone and $365(65 \%)$ of them were reached. Demographic, clinical and therapeutically features were taken from the patients' medical records. Data on clinical course and the disease outcome were collected by using a structured questionnaire which was fulfilled during the phone conversation between investigator and patients parents.

Results: A total of 365 patients with PFAPA were examined: 154 (42\%) of them were female. The mean age at disease onset, at diagnosis and at the investigation was $22.79 \pm 18.8,41.7 \pm 21.7$ and $77.14 \pm 31.35$ months, respectively. The most common disease feature at the disease onset was: recurrent fever in $365(100 \%)$, cryptic tonsillitis in $365(100 \%)$ and aphtous stomatitis in $317(88 \%)$. Sixty three $(17 \%)$ patients met the criteria for both PFAPA and FMF. MEFV gene mutation analysis was performed in $93(25 \%)$ patients and 51 of them $(54 \%)$ had a heterozygous mutation in exon 10. Surgical treatment was performed in 158 $(43 \%)$ patients. Complete clinical remission was achieved in $127(80.3 \%)$ patients. Six $(3 \%)$ showed no response to surgical treatment while $25(15.8 \%)$ patients had a partial response. In patients with partial clinical response, frequency of fever attacks decreased significantly from 17.5 to 7.3 attack per year $(p<0.05)$. Among patients who did not respond to tonsillectomy,11 (52.4\%) were carrier of MEFV heterozygous mutation in exon 10 . There was a statistically significant difference between patients with and without coexistence of FMF features, according to surgical treatment response $(p<0.05)$. The mean age of resolution of PFAPA symptoms in patients who underwent tonsillectomy was $52 \pm 22.4$ months and in patients without tonsillectomy $66 \pm 22.6$ months.

Conclusions: Although PFAPA symptoms usually resolve before age of eight, some patients' complaints persist. FMF should be considered in tonsilloadenoidectomy unresponsive PFAPA patients, especially in endemic regions like Turkey. Tonsilloadenoidectomy seems to be an effective treatment option for pediatric PFAPA patients.

References:

[1] Vanoni F, Theodoropoulou K, Hofer M. PFAPA syndrome: a review on treatment and outcome. Pediatr Rheumatol Online J 2016;14:38

[2] Batu ED, Kara Eroğlu F, Tsoukas P, et al. Periodic Fever, Aphthosis, Pharyngitis, and Adenitis Syndrome: Analysis of Patients From Two Geographic Areas. Arthritis Care Res (Hoboken) 2016:68:1859-1865.

[3] Celiksoy MH, Ogur G, Yaman E, et al. Could familial Mediterranean fever gene mutations be related to PFAPA syndrome? Pediatr Allergy Immunol. 2016;27:78-82.

Disclosure of Interest: None declared

DOI: 10.1136/annrheumdis-2017-eular.4617

\section{THU0499 ANTI-MULLERIAN HORMONE IN A COHORT OF YOUNG ADULT WOMEN WITH JUVENILE IDIOPATHIC ARTHRITIS}

C. Di Mario ${ }^{1}$, M.R. Gigante ${ }^{1}$, A. Musto ${ }^{1}$, A. Barini ${ }^{2}$, A. Barini ${ }^{2}$, B. Tolusso ${ }^{1}$ G. Ferraccioli ${ }^{1}$, E. Gremese ${ }^{1} .{ }^{1}$ Institute of Rheumatology; ${ }^{2}$ Institute of Biochemistry and Clinical Biochemistry, Fondazione Policlinico Universitario A. Gemelli, Catholic University of the Sacred Heart, Rome, Italy

Background: Juvenile Idiopathic Arthritis (JIA) represents one of more common chronic disease of the childhood, affects young people before sixteen and persists into their reproductive years. It is reported in one study that fertility was not impaired in JIA women but potentially it can be compromised by pharmacological treatments such as the prolonged immunosuppressive therapy used in young women patients (1). The Anti-Müllerian Hormone (AMH) is secreted from granulosa ovary cells and serum levels of Anti-Müllerian Hormone are used as a measure of ovarian reserve, reflecting the number of primary follicles. 